\title{
Mixed $g$-monotone property and quadruple fixed point theorems in partially ordered metric spaces
}

\author{
Zead Mustafa1* ${ }^{1 *}$ Hassen Aydi $^{2}$ and Erdal Karapinar ${ }^{3}$
}

\author{
* Correspondence: zmagablh@hu. \\ edu.jo \\ ${ }^{1}$ Department of Mathematics, The \\ Hashemite University, P.O. 330127, \\ Zarqa 13115, Jordan \\ Full list of author information is \\ available at the end of the article
}

\begin{abstract}
In this manuscript, we prove some quadruple coincidence and common fixed point theorems for $F: X^{4} \rightarrow X$ and $g: X \rightarrow X$ satisfying generalized contractions in partially ordered metric spaces. Our results unify, generalize and complement various known results from the current literature. Also, an application to matrix equations is given. 2000 Mathematics subject Classifications: 46T99; 54H25; 47H10; 54E50.
\end{abstract}

Keywords: quadruple coincidence point, quadruple common fixed point, ordered set, metric space, generalized contraction

\section{Introduction and preliminaries}

Existence of fixed points in partially ordered metric spaces was first investigated by Turinici [1], where he extended the Banach contraction principle in partially ordered sets. In 2004, Ran and Reurings [2] presented some applications of Turinici's theorem to matrix equations. Following these initial articles, some remarkable results were reported see, e.g., [3-13].

Gnana Bhashkar and Lakshmikantham in [14] introduced the concept of a coupled fixed point of a mapping $F: X \times X \rightarrow X$ and investigated some coupled fixed point theorems in partially ordered complete metric spaces. Later, Lakshmikantham and Ćirić [15] proved coupled coincidence and coupled common fixed point theorems for nonlinear mappings $F: X \times X \rightarrow X$ and $g: X \rightarrow X$ in partially ordered complete metric spaces. Various results on coupled fixed point have been obtained, since then see, e.g., [6,9,16-33]. Recently, Berinde and Borcut [34] introduced the concept of tripled fixed point in ordered sets.

For simplicity, we denote $\underbrace{X \times X \cdots X \times X}_{k \text { times }}$ by $X^{k}$ where $k \in \mathbb{N}$. Let us recall some basic definitions.

Definition 1.1 (See [34]) Let $(X, \leq)$ be a partially ordered set and $F: X^{3} \rightarrow X$. The mapping $F$ is said to has the mixed monotone property if for any $x, y, z \in X$

$$
\begin{aligned}
& x_{1}, x_{2} \in X, \quad x_{1} \leq x_{2} \Rightarrow F\left(x_{1}, y, z\right) \leq F\left(x_{2}, y, z\right), \\
& y_{1}, y_{2} \in X, \quad y_{1} \leq y_{2} \Rightarrow F\left(x, y_{1}, z\right) \geq F\left(x, y_{2}, z\right) \text {, } \\
& z_{1}, z_{2} \in X, \quad z_{1} \leq z_{2} \Rightarrow F\left(x, y, z_{1}\right) \leq F\left(x, y, z_{2}\right) \text {. }
\end{aligned}
$$

Definition 1.2 Let $F: X^{3} \rightarrow X$. An element $(x, y, z)$ is called a tripled fixed point of $F$ if

(C) 2012 Mustafa et al; licensee Springer. This is an Open Access article distributed under the terms of the Creative Commons Attribution License (http://creativecommons.org/licenses/by/2.0), which permits unrestricted use, distribution, and reproduction in any medium, provided the original work is properly cited. 


$$
F(x, y, z)=x, \quad F(y, x, y)=y \quad \text { and } \quad F(z, y, x)=z .
$$

Also, Berinde and Borcut [34] proved the following theorem:

Theorem 1.1 Let $(X, \leq, d)$ be a partially ordered set and suppose there is a metric $d$ on $X$ such that $(X, d)$ is a complete metric space. Let $F: X^{3} \rightarrow X$ having the mixed monotone property. Suppose there exist $j, r, l \geq 0$ with $j+r+l<1$ such that

$$
d(F(x, y, z), F(u, v, w)) \leq j d(x, u)+r d(y, v)+l d(z, w),
$$

for any $x, y, z \in X$ for which $x \leq u, v \leq y$ and $z \leq w$. Suppose either $F$ is continuous or $X$ has the following properties:

1. if a non-decreasing sequence $x_{n} \rightarrow x$, then $x_{n} \leq x$ for all $n$,

2. if a non-increasing sequence $y_{n} \rightarrow y$, then $y \leq y_{n}$ for all $n$.

If there exist $x_{0}, y_{0}, z_{0} \in X$ such that $x_{0} \leq F\left(x_{0}, y_{0}, z_{0}\right), y_{0} \geq F\left(y_{0}, x_{0}, z_{0}\right)$ and $z_{0} \leq F$ $\left(z_{0}, y_{0}, x_{0}\right)$, then there exist $x, y, z \in X$ such that

$$
F(x, y, z)=x, \quad F(y, x, y)=y \quad \text { and } \quad F(z, y, x)=z,
$$

that is, $F$ has a tripled fixed point.

Recently, Aydi et al. [35] introduced the following concepts.

Definition 1.3 Let $(X, \leq)$ be a partially ordered set. Let $F: X^{3} \rightarrow X$ and $g: X \rightarrow X$. The mapping $F$ is said to has the mixed g-monotone property if for any $x, y, z \in X$

$$
\begin{aligned}
& x_{1}, x_{2} \in X, g x_{1} \leq g x_{2} \Rightarrow F\left(x_{1}, y, z\right) \leq F\left(x_{2}, y, z\right), \\
& y_{1}, y_{2} \in X, g y_{1} \leq g y_{2} \Rightarrow F\left(x, y_{1}, z\right) \geq F\left(x, y_{2}, z\right), \\
& z_{1}, z_{2} \in X, g z_{1} \leq g z_{2} \Rightarrow F\left(x, y, z_{1}\right) \leq F\left(x, y, z_{2}\right) .
\end{aligned}
$$

Definition 1.4 Let $F: X^{3} \rightarrow X$ and $g: X \rightarrow X$. An element $(x, y, z)$ is called a tripled coincidence point of $F$ and $g$ if

$$
F(x, y, z)=g x, \quad F(y, x, y)=g y, \quad \text { and } \quad F(z, y, x)=g z .
$$

( $g x, g y, g z)$ is said a tripled point of coincidence of $F$ and $g$.

Definition 1.5 Let $F: X^{3} \rightarrow X$ and $g: X \rightarrow X$. An element $(x, y, z)$ is called a tripled common fixed point of $F$ and $g$ if

$$
F(x, y, z)=g x=x, \quad F(y, x, y)=g y=y, \quad \text { and } \quad F(z, y, x)=g z=z .
$$

Definition 1.6 Let $X$ be a non-empty set. Then we say that the mappings $F: X^{3} \rightarrow X$ and

$$
\begin{aligned}
& g: X \rightarrow X \text { are commutative if for all } x, y, z \in X \\
& \qquad g(F(x, y, z))=F(g x, g y, g z) .
\end{aligned}
$$

The notion of fixed point of order $N \geq 3$ was first introduced by Samet and Vetro [36]. Very recently, Karapinar used the concept of quadruple fixed point and proved some fixed point theorems on the topic [37]. Following this study, quadruple fixed point is developed and some related fixed point theorems are obtained in [38-41].

Definition 1.7 [38] Let $X$ be a nonempty set and $F: X^{4} \rightarrow X$ be a given mapping. An element $(x, y, z, w) \in X \times X \times X \times X$ is called a quadruple fixed point of $F$ if

$$
F(x, y, z, w)=x, F(y, z, w, x)=y, F(z, w, x, y)=z, \quad \text { and } \quad F(w, x, y, z)=w .
$$


Let $(X, d)$ be a metric space. The mapping $\bar{d}: X^{4} \rightarrow X$, given by

$$
\bar{d}((x, y, z, w),(u, v, h, l))=d(x, y)+d(y, v)+d(z, h)+d(w, l),
$$

defines a metric on $X^{4}$, which will be denoted for convenience by $d$.

Definition 1.8 [38] Let $(X, \leq)$ be a partially ordered set and $F: X^{4} \rightarrow X$ be a mapping. We say that $F$ has the mixed monotone property if $F(x, y, z, w)$ is monotone nondecreasing in $x$ and $z$ and is monotone non-increasing in $y$ and $w$; that is, for any $x, y$, $z, w \in X$,

$$
\begin{array}{cccc}
x_{1}, x_{2} \in X, & x_{1} \leq x_{2} \quad \text { implies } & F\left(x_{1}, y, z, w\right) \leq F\left(x_{2}, y, z, w\right), \\
y_{1}, y_{2} \in X, \quad y_{1} \leq y_{2} \quad \text { implies } & F\left(x, y_{2}, z, w\right) \leq F\left(x, y_{1}, z, w\right), \\
z_{1}, z_{2} \in X, \quad z_{1} \leq z_{2} \quad \text { implies } & F\left(x, y, z_{1}, w\right) \leq F\left(x, y, z_{2}, w\right),
\end{array}
$$

and

$$
w_{1}, w_{2} \in X, \quad w_{1} \leq w_{2} \quad \text { implies } \quad F\left(x, y, z, w_{2}\right) \leq F\left(x, y, z, w_{1}\right) .
$$

In this article, we establish some quadruple coincidence and common fixed point theorems for $F: X^{4} \rightarrow X$ and $g: X \rightarrow X$ satisfying nonlinear contractions in partially ordered metric spaces. Also, some interesting corollaries are derived and an application to matrix equations is given.

\section{Main results}

We start this section with the following definitions.

Definition 2.1 Let $(X, \leq)$ be a partially ordered set. Let $F: X^{4} \rightarrow X$ and $g: X \rightarrow X$. The mapping $F$ is said to has the mixed g-monotone property if for any $x, y, z, w \in X$

$x_{1}, x_{2} \in X, \quad g x_{1} \leq g x_{2} \Rightarrow F\left(x_{1}, y, z, w\right) \leq F\left(x_{2}, y, z, w\right)$,

$y_{1}, y_{2} \in X, \quad g y_{1} \leq g y_{2} \Rightarrow F\left(x, y_{1}, z, w\right) \geq F\left(x, y_{2}, z, w\right)$,

$z_{1}, z_{2} \in X, \quad g z_{1} \leq g z_{2} \Rightarrow F\left(x, y, z_{1}, w\right) \leq F\left(x, y, z_{2}, w\right)$ and

$w_{1}, w_{2} \in X, \quad g w_{1} \leq g w_{2} \Rightarrow F\left(x, y, z, w_{1}\right) \geq F\left(x, y, z, w_{2}\right)$.

Definition 2.2 Let $F: X^{4} \rightarrow X$ and $g: X \rightarrow X$. An element $(x, y, z, w)$ is called a quadruple coincidence point of $F$ and $g$ if

$$
F(x, y, z, w)=g x, \quad F(y, z, w, x)=g y, \quad F(z, w, x, y)=g z, \quad \text { and } \quad F(w, x, y, z)=g w .
$$

$(g x, g y, g z, g w)$ is said a quadruple point of coincidence of $F$ and $g$.

Definition 2.3 Let $F: X^{4} \rightarrow X$ and $g: X \rightarrow X$. An element $(x, y, z, w)$ is called a quadruple common fixed point of $F$ and $g$ if

$$
\begin{aligned}
& F(x, y, z, w)=g x=x, \quad F(y, z, w, x)=g y=y, \\
& F(z, w, x, y)=g z=z, \text { and } F(w, x, y, z)=g w=w .
\end{aligned}
$$

Definition 2.4 Let $X$ be a non-empty set. Then we say that the mappings $F: X^{4} \rightarrow X$ and $g: X \rightarrow \times$ are commutative if for all $x, y, z, w \in X$

$$
g(F(x, y, z, w))=F(g x, g y, g z, g w) .
$$

Let $\Phi$ be the set of all functions $\varphi:[0, \infty) \rightarrow[0, \infty)$ such that:

1. $\varphi(t)<t$ for all $t \in(0,+\infty)$. 
2. $\lim _{r \rightarrow t^{+}} \phi(r)<t$ for all $t \in(0,+\infty)$.

For simplicity, we define the following.

$$
M(x, y, z, w, u, v, h, l)=\min \left\{\begin{array}{c}
d(F(x, y, z, w), g x), d(F(x, y, z, w), g u), \\
d(F(u, v, h, l), g u)
\end{array}\right\} .
$$

Now, we state the first main result of this article.

Theorem 2.1 Let $(X, \leq)$ be a partially ordered set and suppose there is a metric $d$ on $X$ such that $(X, d)$ is a complete metric space. Suppose $F: X^{4} \rightarrow X$ and $g: X \rightarrow X$ are such that $F$ is continuous and has the mixed g-monotone property. Assume also that there exist $\varphi \in \Phi$ and $L \geq 0$ such that

$$
\begin{aligned}
d(F(x, y, z, w), F(u, v, h, l)) \leq & \phi(\max \{d(g x, g u), d(g y, g v), d(g z, g h), d(g w, g l)\}) \\
& +L M(x, y, z, w, u, v, h, l)
\end{aligned}
$$

for any $x, y, z, w, u, v, h, l \in X$ for which $g x \leq g u, g v \leq g y, g z \leq g h$ and $g l \leq g w$. Suppose $F\left(X^{4}\right) \subset g(X), g$ is continuous and commutes with $F$. If there exist $x_{0}, y_{0}, z_{0}, w_{0} \in$ $X$ such that

$$
\begin{aligned}
& g x_{0} \leq F\left(x_{0}, y_{0}, z_{0}, w_{0}\right), \\
& g z_{0} \leq F\left(z_{0}, w_{0}, x_{0}, y_{0}\right), \quad \text { and } \quad g w_{0} \geq F\left(w_{0}, x_{0}, y_{0}, z_{0}\right),
\end{aligned}
$$

then there exist $x, y, z, w \in X$ such that

$$
F(x, y, z, w)=g x, \quad F(y, z, w, x)=g y, \quad F(z, w, x, y)=g z \text { and } F(w, x, y, z)=g w
$$

that is, $F$ and $g$ have a quadruple coincidence point.

Proof. Let $x_{0}, y_{0}, z_{0}, w_{0} \in X$ such that

$$
\begin{aligned}
& g x_{0} \leq F\left(x_{0}, y_{0}, z_{0}, w_{0}\right), \quad g y_{0} \geq F\left(y_{0}, z_{0}, w_{0}, x_{0}\right), \\
& g z_{0} \leq F\left(z_{0}, w_{0}, x_{0}, y_{0}\right) \text { and } g w_{0} \geq F\left(w_{0}, x_{0}, y_{0}, z_{0}\right) .
\end{aligned}
$$

Since $F\left(X^{4}\right) \subset g(X)$, then we can choose $x_{1}, y_{1}, z_{1}, w_{1} \in X$ such that

$$
\begin{aligned}
& g x_{1}=F\left(x_{0}, y_{0}, z_{0}, w_{0}\right), \\
& g z_{1}=F\left(z_{0}, w_{0}, x_{0}, y_{0}\right)
\end{aligned} \quad \begin{aligned}
g y_{1} & =F\left(y_{0}, z_{0}, w_{0}, x_{0}\right), \\
g w_{1} & =F\left(w_{0}, x_{0}, y_{0}, z_{0}\right) .
\end{aligned}
$$

Taking into account $F\left(X^{4}\right) \subset g(X)$, by continuing this process, we can construct sequences $\left\{x_{n}\right\},\left\{y_{n}\right\},\left\{z_{n}\right\}$, and $\left\{w_{n}\right\}$ in $X$ such that

$$
\begin{aligned}
& g x_{n+1}=F\left(x_{n}, y_{n}, z_{n}, w_{n}\right), \\
& g z_{n+1}=F\left(z_{n}, w_{n}, x_{n}, y_{n}\right), \quad \text { and } \quad g y_{n+1}=F\left(y_{n}, z_{n}, w_{n}, x_{n}\right),
\end{aligned}
$$

We shall show that

$$
g x_{n} \leq g x_{n+1}, \quad g y_{n+1} \leq g y_{n}, \quad g z_{n} \leq g z_{n+1}, \quad \text { and } g w_{n+1} \leq g w_{n} \text { for } n=0,1,2, \ldots
$$

For this purpose, we use the mathematical induction. Since, $g x_{0} \leq F\left(x_{0}, y_{0}, z_{0}, w_{0}\right)$, $g y_{0} \geq F\left(y_{0}, z_{0}, w_{0}, x_{0}\right), g z_{0} \leq F\left(z_{0}, w_{0}, x_{0}, y_{0}\right)$, and $g w_{0} \geq F\left(w_{0}, x_{0}, y_{0}, z_{0}\right)$, then by (4), we get

$$
g x_{0} \leq g x_{1}, \quad g y_{1} \leq g y_{0}, \quad g z_{0} \leq g z_{1}, \quad \text { and } g w_{1} \leq g w_{0}
$$


that is, (6) holds for $n=0$.

We presume that (6) holds for some $n>0$. As $F$ has the mixed $g$-monotone property and $g x_{n} \leq g x_{n+1}, g y_{n+1} \leq g y_{n}, g z_{n} \leq g z_{n+1}$ and $g w_{n+1} \leq g w_{n}$, we obtain

$$
\begin{aligned}
g x_{n+1} & =F\left(x_{n}, y_{n}, z_{n}, w_{n}\right) \leq F\left(x_{n+1}, y_{n}, z_{n}, w_{n}\right) \\
& \leq F\left(x_{n+1}, y_{n}, z_{n+1}, w_{n}\right) \leq F\left(x_{n+1}, y_{n+1}, z_{n+1}, w_{n}\right) \\
& \leq F\left(x_{n+1}, y_{n+1}, z_{n+1}, w_{n+1}\right)=g x_{n+2}, \\
g y_{n+2} & =F\left(y_{n+1}, z_{n+1}, w_{n+1}, x_{n+1}\right) \leq F\left(y_{n+1}, z_{n}, x_{n+1}, w_{n+1}\right) \\
& \leq F\left(y_{n}, z_{n}, x_{n+1}, w_{n+1}\right) \leq F\left(y_{n}, z_{n}, x_{n}, w_{n+1}\right) \\
& \leq F\left(y_{n}, z_{n}, x_{n}, w_{n}\right)=g y_{n+1}, \\
g z_{n+1} & =F\left(z_{n}, y_{n}, x_{n}, w_{n}\right) \leq F\left(z_{n+1}, y_{n}, x_{n}, w_{n}\right) \\
& \leq F\left(z_{n+1}, y_{n+1}, x_{n}, w_{n}\right) \leq F\left(z_{n+1}, y_{n+1}, x_{n+1}, w_{n}\right) \\
& \leq F\left(z_{n+1}, y_{n+1}, x_{n+1}, w_{n+1}\right)=g z_{n+2},
\end{aligned}
$$

and

$$
\begin{aligned}
g w_{n+2} & =F\left(w_{n+1}, x_{n+1}, y_{n+1}, z_{n+1}\right) \leq F\left(w_{n+1}, x_{n}, y_{n+1}, z_{n+1}\right) \\
& \leq F\left(w_{n}, x_{n}, y_{n+1}, z_{n+1}\right) \leq F\left(w_{n}, x_{n}, y_{n}, z_{n+1}\right) \\
& \leq F\left(w_{n}, x_{n}, y_{n}, z_{n}\right)=g w_{n+1} .
\end{aligned}
$$

Thus, (6) holds for any $n \in \mathbb{N}$. Assume for some $n \in \mathbb{N}$,

$$
g x_{n}=g x_{n+1}, \quad g y_{n}=g y_{n+1}, \quad g z_{n}=g z_{n+1}, \quad \text { and } \quad g w_{n}=g w_{n+1}
$$

then, by (5), $\left(x_{n}, y_{n}, z_{n}, w_{n}\right)$ is a quadruple coincidence point of $F$ and $g$. From now on, assume for any $n \in \mathbb{N}$ that at least

$$
g x_{n} \neq g x_{n+1} \quad \text { or } \quad g y_{n} \neq g y_{n+1} \quad \text { or } \quad g z_{n} \neq g z_{n+1} \quad \text { or } \quad g w_{n} \neq g w_{n+1} .
$$

By (2) and (5), it is easy that

$$
\begin{aligned}
M\left(x_{n-1}, y_{n-1}, z_{n-1}, w_{n-1}, x_{n}, y_{n}, z_{n}, w_{n}\right)=M\left(y_{n}, z_{n}, w_{n}, x_{n}, y_{n-1}, z_{n-1}, w_{n-1}, x_{n-1}\right) \\
=M\left(z_{n-1}, y_{n-1}, x_{n-1}, z_{n}, y_{n}, x_{n}\right) \\
=M\left(w_{n}, x_{n}, y_{n}, z_{n}, w_{n-1}, x_{n-1}, y_{n-1}, z_{n-1}\right)=0 \text { for all } n \geq 1 .
\end{aligned}
$$

Due to (3) and (8), we have

$$
\begin{aligned}
& d\left(g x_{n}, g x_{n+1}\right)=d\left(F\left(x_{n-1}, y_{n-1}, z_{n-1}, w_{n-1}\right), F\left(x_{n}, y_{n}, z_{n}, w_{n}\right)\right) \\
& \leq \phi\left(\max \left\{d\left(g x_{n-1}, g x_{n}\right), d\left(g y_{n-1}, g y_{n}\right), d\left(g z_{n-1}, g z_{n}\right), d\left(g w_{n-1}, g w_{n}\right)\right\}\right) \\
& +L M\left(x_{n-1}, y_{n-1}, z_{n-1}, w_{n-1}, x_{n}, y_{n}, z_{n}, w_{n}\right) \\
& =\phi\left(\max \left\{d\left(g x_{n-1}, g x_{n}\right), d\left(g y_{n-1}, g y_{n}\right), d\left(g z_{n-1}, g z_{n}\right), d\left(g w_{n-1}, g w_{n}\right)\right\}\right), \\
& d\left(g y_{n}, g y_{n+1}\right)=d\left(F\left(y_{n}, z_{n}, w_{n}, x_{n}\right), y_{n-1}, F\left(y_{n-1}, z_{n-1}, w_{n-1}, x_{n-1}\right)\right) \\
& \leq \phi\left(\max \left\{d\left(g y_{n-1}, g y_{n}\right), d\left(g x_{n-1}, g x_{n}\right), d\left(g z_{n-1}, g z_{n}\right), d\left(g w_{n-1}, g w_{n}\right)\right\}\right), \\
& +L M\left(y_{n}, z_{n}, w_{n}, w_{n}, y_{n-1}, z_{n-1}, w_{n-1}, x_{n-1}\right) \\
& =\phi\left(\max \left\{d\left(g y_{n-1}, g y_{n}\right), d\left(g x_{n-1}, g x_{n}\right), d\left(g z_{n-1}, g z_{n}\right), d\left(g w_{n-1}, g w_{n}\right)\right\}\right),
\end{aligned}
$$




$$
\begin{aligned}
& d\left(g z_{n}, g z_{n+1}\right)=d\left(F\left(z_{n-1}, w_{n-1}, x_{n-1}, y_{n-1}\right), F\left(z_{n}, w_{n}, x_{n}, y_{n}\right)\right) \\
& \leq \phi\left(\max \left\{, d\left(g z_{n-1}, g z_{n}\right), d\left(g w_{n-1}, g w_{n}\right), d\left(g x_{n-1}, g x_{n}\right), d\left(g y_{n-1}, g y_{n}\right)\right\}\right) \\
& +L M\left(z_{n-1}, w_{n-1}, x_{n-1}, y_{n-1}, z_{n}, w_{n}, x_{n}, y_{n}\right) \\
& =\phi\left(\max \left\{d\left(g z_{n-1}, g z_{n}\right), d\left(g w_{n-1}, g w_{n}\right), d\left(g x_{n-1}, g x_{n}\right), d\left(g y_{n-1}, g y_{n}\right)\right\}\right)
\end{aligned}
$$

and

$$
\begin{aligned}
& d\left(g w_{n}, g w_{n+1}\right)=d\left(F\left(w_{n}, x_{n}, y_{n}, z_{n}\right), F\left(w_{n-1}, x_{n-1}, y_{n-1}, z_{n-1}\right)\right) \\
& \leq \phi\left(\max \left\{d\left(g w_{n-1}, g w_{n}\right), d\left(g x_{n-1}, g x_{n}\right), d\left(g y_{n-1}, g y_{n}\right), d\left(g z_{n-1}, g z_{n}\right)\right\}\right), \\
& +L M\left(w_{n}, x_{n}, y_{n}, z_{n}, w_{n-1}, x_{n-1}, y_{n-1}, z_{n-1}\right) \\
& =\phi\left(\max \left\{d\left(g w_{n-1}, g w_{n}\right), d\left(g x_{n-1}, g x_{n}\right), d\left(g y_{n-1}, g y_{n}\right), d\left(g z_{n-1}, g z_{n}\right)\right\}\right) .
\end{aligned}
$$

Having in mind that $\varphi(t)<t$ for all $t>0$, so from (9)-(12) we obtain that

$$
\begin{aligned}
0 & <\max \left\{d\left(g x_{n}, g x_{n+1}\right), d\left(g y_{n}, g y_{n+1}\right), d\left(g z_{n}, g z_{n+1}\right), d\left(g w_{n}, g w_{n+1}\right)\right\} \\
& \leq \phi\left(\max \left\{d\left(g z_{n-1}, g z_{n}\right), d\left(g y_{n-1}, g y_{n}\right), d\left(g x_{n-1}, g x_{n}\right), d\left(g w_{n-1}, g w_{n}\right)\right\}\right) \\
& <\max \left\{d\left(g z_{n-1}, g z_{n}\right), d\left(g y_{n-1}, g y_{n}\right), d\left(g x_{n-1}, g x_{n}\right), d\left(g w_{n-1}, g w_{n}\right)\right\} .
\end{aligned}
$$

It follows that

$$
\max \left\{\begin{array}{c}
d\left(g x_{n}, g x_{n+1}\right), d\left(g y_{n}, g y_{n+1},\right. \\
d\left(g z_{n}, g z_{n+1}\right), d\left(g w_{n}, g w_{n+1}\right)
\end{array}\right\}<\max \left\{\begin{array}{c}
d\left(g z_{n-1}, g z_{n}\right), d\left(g y_{n-1}, g y_{n}\right), \\
d\left(g x_{n-1}, g x_{n}\right), d\left(g w_{n-1}, g w_{n}\right)
\end{array}\right\} .
$$

Thus, $\left\{\max \left\{d\left(g x_{n}, g x_{n+1}\right), d\left(g y_{n}, g y_{n+1}\right), d\left(g z_{n}, g z_{n+1}\right), d\left(g w_{n}, g w_{n+1}\right)\right\}\right\}$ is a positive decreasing sequence. Hence, there exists $r \geq 0$ such that

$$
\lim _{n \rightarrow+\infty} \max \left\{d\left(g x_{n}, g x_{n+1}\right), d\left(g y_{n}, g y_{n+1}\right), d\left(g z_{n}, g z_{n+1}\right), d\left(g w_{n}, g w_{n+1}\right)\right\}=r .
$$

Suppose that $r>0$. Letting $n \rightarrow+\infty$ in (13), we obtain that

$$
0<r \leq \lim _{n \rightarrow+\infty} \phi\left(\max \left\{\begin{array}{c}
d\left(g z_{n-1}, g z_{n}\right), d\left(g y_{n-1}, g y_{n}\right), \\
d\left(g x_{n-1}, g x_{n}\right), d\left(g w_{n-1}, g w_{n}\right)
\end{array}\right\}\right)=\lim _{t \rightarrow r^{+}} \phi(t)<r .
$$

It is a contradiction. We deduce that

$$
\lim _{n \rightarrow+\infty} \max \left\{d\left(g x_{n}, g x_{n+1}\right), d\left(g y_{n}, g y_{n+1}\right), d\left(g z_{n}, g z_{n+1}\right), d\left(g w_{n}, g w_{n+1}\right)\right\}=0 .
$$

We shall show that $\left\{g x_{n}\right\},\left\{g y_{n}\right\},\left\{g z_{n}\right\}$, and $\left\{g w_{n}\right\}$ are Cauchy sequences in the metric space $(X, d)$. Assume the contrary, that is, one of the sequence $\left\{g x_{n}\right\},\left\{g y_{n}\right\},\left\{g z_{n}\right\}$ or $\left\{g w_{n}\right\}$ is not a Cauchy, that is,

$$
\lim _{n, m \rightarrow+\infty} d\left(g x_{m}, g x_{n}\right) \neq 0 \quad \text { or } \quad \lim _{n, m \rightarrow+\infty} d\left(g y_{m}, g y_{n}\right) \neq 0
$$

or

$$
\lim _{n, m \rightarrow+\infty} d\left(g z_{m}, g z_{n}\right) \neq 0 \text { or } \lim _{n, m \rightarrow+\infty} d\left(g w_{m}, g w_{n}\right) \neq 0 .
$$

This means that there exists $\varepsilon>0$, for which we can find subsequences of integers $\left(m_{k}\right)$ and $\left(n_{k}\right)$ with $n_{k}>m_{k}>k$ such that

$$
\max \left\{d\left(g x_{m_{k}}, g x_{n_{k}}\right), d\left(g y_{m_{k}}, g y_{n_{k}}\right), d\left(g z_{m_{k}}, g z_{n_{k}}\right), d\left(g w_{m_{k}}, g w_{n_{k}}\right)\right\} \geq \varepsilon .
$$


Further, corresponding to $m_{k}$ we can choose $n_{k}$ in such a way that it is the smallest integer with $n_{k}>m_{k}$ and satisfying (17). Then

$$
\max \left\{d\left(g x_{m_{k}}, g x_{n_{k}-1}\right), d\left(g y_{m_{k}}, g y_{n_{k}-1}\right), d\left(g z_{m_{k}}, g z_{n_{k}-1}\right), d\left(g w_{m_{k}}, g w_{n_{k}-1}\right)\right\}<\varepsilon .
$$

By triangular inequality and (18), we have

$$
\begin{aligned}
d\left(g x_{m_{k}}, g x_{n_{k}}\right) & \leq d\left(g x_{m_{k}}, g x_{n_{k}-1}\right)+d\left(g x_{n_{k}-1}, g x_{n_{k}}\right) \\
& <\varepsilon+d\left(g x_{n_{k}-1}, g x_{n_{k}}\right) .
\end{aligned}
$$

Thus, by (16) we obtain

$$
\lim _{k \rightarrow+\infty} d\left(g x_{m_{k}}, g x_{n_{k}}\right) \leq \lim _{k \rightarrow+\infty} d\left(g x_{m_{k}}, g x_{n_{k}-1}\right) \leq \varepsilon .
$$

Similarly, we have

$$
\begin{aligned}
& \lim _{k \rightarrow+\infty} d\left(g y_{m_{k}}, g y_{n_{k}}\right) \leq \lim _{k \rightarrow+\infty} d\left(g y_{m_{k}}, g y_{n_{k}-1}\right) \leq \varepsilon, \\
& \lim _{k \rightarrow+\infty} d\left(g z_{m_{k}}, g z_{n_{k}}\right) \leq \lim _{k \rightarrow+\infty} d\left(g z_{m_{k}}, g z_{n_{k}-1}\right) \leq \varepsilon,
\end{aligned}
$$

and

$$
\lim _{k \rightarrow+\infty} d\left(g w_{m_{k}}, g w_{n_{k}}\right) \leq \lim _{k \rightarrow+\infty} d\left(g w_{m_{k}}, g w_{n_{k}-1}\right) \leq \varepsilon .
$$

Again by (18), we have

$$
\begin{aligned}
d\left(g x_{m_{k}}, g x_{n_{k}}\right) \leq & d\left(g x_{m_{k}}, g x_{m_{k}-1}\right)+d\left(g x_{m_{k}-1}, g x_{n_{k}-1}\right)+d\left(g x_{n_{k}-1}, g x_{n_{k}}\right) \\
\leq & d\left(g x_{m_{k}}, g x_{m_{k}-1}\right)+d\left(g x_{m_{k}-1}, g x_{m_{k}}\right) \\
& +d\left(g x_{m_{k}}, g x_{n_{k}-1}\right)+d\left(g x_{n_{k}-1}, g x_{n_{k}}\right) \\
< & d\left(g x_{m_{k}}, g x_{m_{k}-1}\right)+d\left(g x_{m_{k}-1}, g x_{m_{k}}\right)+\varepsilon+d\left(g x_{n_{k}-1}, g x_{n_{k}}\right) .
\end{aligned}
$$

Letting $k \rightarrow+\infty$ and using (16), we get

$$
\begin{aligned}
& \lim _{k \rightarrow+\infty} d\left(g x_{m_{k}}, g x_{n_{k}}\right) \leq \lim _{k \rightarrow+\infty} d\left(g x_{m_{k}-1}, g x_{n_{k}-1}\right) \leq \varepsilon \\
& \lim _{k \rightarrow+\infty} d\left(g y_{m_{k}}, g y_{n_{k}}\right) \leq \lim _{k \rightarrow+\infty} d\left(g y_{m_{k}-1}, g y_{n_{k}-1}\right) \leq \varepsilon \\
& \lim _{k \rightarrow+\infty} d\left(g z_{m_{k}}, g z_{n_{k}}\right) \leq \lim _{k \rightarrow+\infty} d\left(g z_{m_{k}-1}, g z_{n_{k}-1}\right) \leq \varepsilon
\end{aligned}
$$

and

$$
\lim _{k \rightarrow+\infty} d\left(g w_{m_{k}}, g w_{n_{k}}\right) \leq \lim _{k \rightarrow+\infty} d\left(g w_{m_{k}-1}, g w_{n_{k}-1}\right) \leq \varepsilon .
$$

Using (17) and (23)-(26), we have

$$
\begin{aligned}
& \lim _{k \rightarrow+\infty} \max \left\{d\left(g x_{m_{k}}, g x_{n_{k}}\right), d\left(g y_{m_{k}}, g y_{n_{k}}\right), d\left(g z_{m_{k}}, g z_{n_{k}}\right), d\left(g w_{m_{k}}, g w_{n_{k}}\right)\right\} \\
& =\lim _{k \rightarrow+\infty} \max \left\{d\left(g x_{m_{k}-1}, g x_{n_{k}-1}\right), d\left(g y_{m_{k}-1}, g y_{n_{k}-1}\right), d\left(g z_{m_{k}-1}, g z_{n_{k}-1}\right), d\left(g w_{m_{k}-1}, g w_{n_{k}-1}\right)\right\} \\
& =\varepsilon .
\end{aligned}
$$


By (16), it is easy to see that

$$
\begin{aligned}
& \lim _{k \rightarrow+\infty} M\left(x_{m_{k}-1}, y_{m_{k}-1}, z_{m_{k}-1}, w_{m_{k}-1}, x_{n_{k}-1}, y_{n_{k}-1}, z_{n_{k}-1}, w_{n_{k}-1}\right) \\
& =\lim _{k \rightarrow+\infty} M\left(y_{n_{k}-1}, z_{n_{k}-1}, w_{n_{k}-1}, x_{n_{k}-1}, y_{m_{k}-1}, z_{m_{k}-1}, w_{m_{k}-1}, x_{m_{k}-1}\right) \\
& =\lim _{k \rightarrow+\infty} M\left(z_{m_{k}-1}, w_{m_{k}-1}, x_{m_{k}-1}, y_{m_{k}-1}, z_{n_{k}-1}, w_{n_{k}-1}, x_{n_{k}-1}, y_{m_{k}-1}\right) \\
& =\lim _{k \rightarrow+\infty} M\left(w_{n_{k}-1}, x_{n_{k}-1}, y_{m_{k}-1}, z_{n_{k}-1}, w_{m_{k}-1}, x_{m_{k}-1}, y_{m_{k}-1}, z_{m_{k}-1}\right)=0
\end{aligned}
$$

Now, using inequality (3), we obtain

$$
\begin{aligned}
d\left(g x_{m_{k}}, g x_{n_{k}}\right) & =d\left(F\left(x_{m_{k}-1}, y_{m_{k}-1}, z_{m_{k}-1}, w_{m_{k}-1}\right), F\left(x_{n_{k}-1}, y_{n_{k}-1}, z_{n_{k}-1}, w_{n_{k}-1}\right)\right) \\
\leq & \phi\left(\max \left\{d\left(x_{m_{k}-1}, x_{n_{k}-1}\right), d\left(y_{m_{k}-1}, y_{n_{k}-1}\right), d\left(z_{m_{k}-1}, z_{n_{k}-1}\right), d\left(w_{m_{k}-1}, w_{n_{k}-1}\right)\right\}\right) \\
+ & L M\left(x_{m_{k}-1}, y_{m_{k}-1}, z_{m_{k}-1}, w_{m_{k}-1}, x_{n_{k}-1}, y_{n_{k}-1}, z_{n_{k}-1}, w_{n_{k}-1}\right), \\
d\left(g y_{n_{k}}, g y_{m_{k}}\right) & =d\left(F\left(y_{n_{k}-1}, z_{n_{k}-1}, w_{n_{k}-1}, x_{n_{k}-1}\right), F\left(y_{m_{k}-1}, z_{m_{k}-1}, w_{m_{k}-1}, x_{m_{k}-1}\right)\right) \\
\leq & \phi\left(\max \left\{d\left(y_{m_{k}-1}, y_{n_{k}-1}\right), d\left(z_{m_{k}-1}, z_{n_{k}-1}\right), d\left(w_{m_{k}-1}, w_{n_{k}-1}, d\left(x_{m_{k}-1}, x_{n_{k}-1}\right)\right\}\right)\right. \\
+ & L M\left(y_{n_{k}-1}, z_{n_{k}-1}, w_{n_{k}-1}, x_{n_{k}-1}, y_{m_{k}-1}, z_{m_{k}-1}, w_{m_{k}-1}, x_{m_{k}-1}\right), \\
d\left(g z_{m_{k}}, g z_{n_{k}}\right) & =d\left(F\left(z_{m_{k}-1}, w_{m_{k}-1}, x_{m_{k}-1}, y_{m_{k}-1}\right), F\left(z_{n_{k}-1}, w_{n_{k}-1}, x_{n_{k}-1}, y_{n_{k}-1}\right)\right) \\
\leq & \phi\left(\max \left\{d\left(z_{m_{k}-1}, z_{n_{k}-1}\right), d\left(w_{m_{k}-1}, w_{n_{k}-1}, d\left(x_{m_{k}-1}, x_{n_{k}-1}\right), d\left(y_{m_{k}-1}, y_{n_{k}-1}\right)\right\}\right)\right. \\
+ & L M\left(z_{m_{k}-1}, w_{m_{k}-1}, x_{m_{k}-1}, y_{m_{k}-1}, z_{n_{k}-1}, w_{n_{k}-1}, x_{n_{k}-1}, y_{m_{k}-1}\right)
\end{aligned}
$$

and

$$
\begin{aligned}
& d\left(g w_{n_{k}}, g w_{m_{k}}\right)=d\left(F\left(w_{n_{k}-1}, x_{n_{k}-1}, y_{n_{k}-1}, z_{n_{k}-1}\right), F\left(w_{m_{k}-1}, x_{m_{k}-1}, y_{m_{k}-1}, z_{m_{k}-1}\right)\right) \\
& \quad \leq \phi\left(\max \left\{d\left(w_{m_{k}-1}, w_{n_{k}-1}, d\left(x_{m_{k}-1}, x_{n_{k}-1}\right), d\left(y_{m_{k}-1}, y_{n_{k}-1}\right), d\left(z_{m_{k}-1}, z_{n_{k}-1}\right)\right\}\right)\right. \\
& +L M\left(w_{n_{k}-1}, x_{n_{k}-1}, y_{n_{k}-1}, z_{n_{k}-1}, w_{m_{k}-1}, x_{m_{k}-1}, y_{m_{k}-1}, z_{m_{k}-1}\right) .
\end{aligned}
$$

From (29)-(32), we deduce that

$$
\begin{aligned}
& \max \left\{d\left(g x_{m_{k}}, g x_{n_{k}}\right), d\left(g y_{m_{k}}, g y_{n_{k}}\right), d\left(g z_{m_{k}}, g z_{n_{k}}\right), d\left(g w_{m_{k}}, g w_{n_{k}}\right)\right\} \\
& \leq \phi\left(\max \left\{d\left(x_{m_{k}-1}, x_{n_{k}-1}\right), d\left(y_{m_{k}-1}, y_{n_{k}-1}\right), d\left(z_{m_{k}-1}, z_{n_{k}-1}\right), d\left(g w_{m_{k}}, g w_{n_{k}}\right)\right\}\right) \\
& +L M\left(x_{m_{k}-1}, y_{m_{k}-1}, z_{m_{k}-1}, w_{m_{k}-1}, x_{n_{k}-1}, y_{n_{k}-1}, z_{n_{k}-1}, w_{n_{k}-1}\right) \\
& +L M\left(y_{n_{k}-1}, z_{n_{k}-1}, w_{n_{k}-1}, x_{n_{k}-1}, y_{m_{k}-1}, z_{m_{k}-1}, w_{m_{k}-1}, x_{m_{k}-1}\right) \\
& +L M\left(z_{m_{k}-1}, w_{m_{k}-1}, x_{m_{k}-1}, y_{m_{k}-1}, z_{n_{k}-1}, w_{n_{k}-1}, x_{n_{k}-1}, y_{m_{k}-1}\right) \\
& +L M\left(w_{n_{k}-1}, x_{n_{k}-1}, y_{n_{k}-1}, z_{n_{k}-1}, w_{m_{k}-1}, x_{m_{k}-1}, y_{m_{k}-1}, z_{m_{k}-1}\right) .
\end{aligned}
$$

Letting $k \rightarrow+\infty$ in (33) and having in mind (27) and (28), we get that

$$
0<\varepsilon \leq \lim _{t \rightarrow \varepsilon^{+}} \phi(t)<\varepsilon,
$$

it is a contradiction. Thus, $\left\{g x_{n}\right\},\left\{g y_{n}\right\},\left\{g z_{n}\right\}$, and $\left\{g w_{n}\right\}$ are Cauchy sequences in $(X$, d).

Since $(X, d)$ is complete, there exist $x, y, z, w \in X$ such that

$$
\lim _{n \rightarrow+\infty} g x_{n}=x, \quad \lim _{n \rightarrow+\infty} g y_{n}=y, \lim _{n \rightarrow+\infty} g y_{n}=y, \quad \text { and } \quad \lim _{n \rightarrow+\infty} g w_{n}=w
$$

From (34) and the continuity of $g$, we have

$$
\lim _{n \rightarrow+\infty} g\left(g x_{n}\right)=g x, \quad \lim _{n \rightarrow+\infty} g\left(g y_{n}\right)=g y, \quad \lim _{n \rightarrow+\infty} g\left(g z_{n}\right)=g z, \quad \text { and } \quad \lim _{n \rightarrow+\infty} g\left(g w_{n}\right)=g w .
$$


From (5) and the commutativity of $F$ and $g$, we have

$$
\begin{aligned}
& g\left(g x_{n+1}\right)=g\left(F\left(x_{n}, y_{n}, z_{n}, w_{n}\right)\right)=F\left(g x_{n}, g y_{n}, g z_{n}, g w_{n}\right), \\
& g\left(g y_{n+1}\right)=g\left(F\left(y_{n}, z_{n}, w_{n}, x_{n}\right)\right)=F\left(g y_{n}, g z_{n}, g w_{n}, g x_{n}\right), \\
& g\left(g z_{n+1}\right)=g\left(F\left(z_{n}, w_{n}, x_{n}, y_{n}\right)\right)=F\left(g z_{n}, g w_{n}, g x_{n}, y_{n}\right),
\end{aligned}
$$

and

$$
g\left(g w_{n+1}\right)=g\left(F\left(w_{n}, x_{n}, y_{n}, z_{n}\right)\right)=F\left(g w_{n}, g x_{n}, y_{n}, g z_{n}\right) .
$$

Now we shall show that $g x=F(x, y, z, w), g y=F(y, z, w, x), g z=F(z, w, x, y)$, and $g w=F(w, x, y, z)$.

By letting $n \rightarrow+\infty$ in (36) - (39), by (34), (35) and the continuity of $F$, we obtain

$$
\begin{aligned}
g x & =\lim _{n \rightarrow+\infty} g\left(g x_{n+1}\right)=\lim _{n \rightarrow+\infty} F\left(g x_{n}, g y_{n}, g z_{n}, g w_{n}\right) \\
& =F\left(\lim _{n \rightarrow+\infty} g x_{n}, \lim _{n \rightarrow+\infty} g y_{n}, \lim _{n \rightarrow+\infty} g z_{n}, \lim _{n \rightarrow+\infty} g w_{n}\right) \\
& =F(x, y, z, w), \\
g y & =\lim _{n \rightarrow+\infty} g\left(g y_{n+1}\right)=\lim _{n \rightarrow+\infty} F\left(g y_{n}, g z_{n}, g w_{n}, g x_{n}\right) \\
& =F\left(\lim _{n \rightarrow+\infty} g y_{n,} \lim _{n \rightarrow+\infty} g z_{n}, \lim _{n \rightarrow+\infty} g w_{n}, \lim _{n \rightarrow+\infty} g w_{n}\right) \\
& =F(y, z, w, x), \\
g z & =\lim _{n \rightarrow+\infty} g\left(g z_{n+1}\right)=\lim _{n \rightarrow+\infty} F\left(g z_{n}, g w_{n}, g x_{n}, g y_{n}\right) \\
& =F\left(\lim _{n \rightarrow+\infty} g z_{n}, \lim _{n \rightarrow+\infty} g w_{n}, \lim _{n \rightarrow+\infty} g x_{n}, \lim _{n \rightarrow+\infty} g y_{n}\right) \\
& =F(z, w, x, y),
\end{aligned}
$$

and

$$
\begin{aligned}
g w & =\lim _{n \rightarrow+\infty} g\left(g w_{n+1}\right)=\lim _{n \rightarrow+\infty} F\left(g w_{n}, g x_{n}, g y_{n}, g z_{n}\right) \\
& =F\left(\lim _{n \rightarrow+\infty} g w_{n}, \lim _{n \rightarrow+\infty} g x_{n}, \lim _{n \rightarrow+\infty} g y_{n}, \lim _{n \rightarrow+\infty} g z_{n}\right) \\
& =F(w, x, y, z) .
\end{aligned}
$$

We have proved that $F$ and $g$ have a quadruple coincidence point. This completes the proof of Theorem 2.1.

In the following theorem, we omit the continuity hypothesis of $F$. We need the following definition.

Definition 2.5 Let $(X, \leq)$ be a partially ordered metric set and $d$ be a metric on $X$. We say that $(X, d, \leq)$ is regular if the following conditions hold:

(i) if non-decreasing sequence $a_{n} \rightarrow a$, then $a_{n} \leq a$ for all $n$,

(ii) if non-increasing sequence $b_{n} \rightarrow b$, then $b \leq b_{n}$ for all $n$.

Theorem 2.2 Let $(X, \leq)$ be a partially ordered set and $d$ be a metric on $X$ such that $(X, d, \leq)$ is regular. Suppose $F: X^{4} \rightarrow X$ and $g: X \rightarrow X$ are such that $F$ has the mixed $g$-monotone property. Assume that there exist $\varphi \in \Phi$ and $L \geq 0$ such that 


$$
\begin{aligned}
d(F(x, y, z, w), F(u, v,, h, l)) & \leq \phi(\max \{d(g x, g u), d(g y, g v), d(g z, g h), d(g w, g l)\}) \\
& +L M(x, y, z, w, u, v, h, l)
\end{aligned}
$$

for any $x, y, z, w, u, v, h, l \in X$ for which $g x \leq g u, g v \leq g y, g z \leq g h$, and $g l \leq g w$. Also, suppose $F\left(X^{4}\right) \subset g(X)$ and $(g(X), d)$ is a complete metric space. If there exist $x_{0}, y_{0}, z_{0}$, $w_{0} \in X$ such that $g x_{0} \leq F\left(x_{0}, y_{0}, z_{0}, w_{0}\right), g y_{0} \geq F\left(y_{0}, z_{0}, w_{0}, x_{0}\right), g z_{0} \leq F\left(z_{0}, w_{0}, x_{0}, y_{0}\right)$ and $g w_{0} \geq F\left(w_{0}, x_{0}, y_{0}, z_{0}\right)$, then there exist $x, y, z, w \in X$ such that

$$
F(x, y, z, w)=g x, \quad F(y, z, w, x)=g y, \quad F(z, w, x, y)=g z \text { and } F(w, x, y, z)=g w
$$

that is, $F$ and $g$ have a quadruple coincidence point.

Proof. Proceeding exactly as in Theorem 2.1, we have that $\left\{g x_{n}\right\},\left\{g y_{n}\right\},\left\{g z_{n}\right\}$, and $\left\{g w_{n}\right\}$ are Cauchy sequences in the complete metric space $(g(X), d)$. Then, there exist $x$, $y, z, w \in X$ such that

$$
g x_{n} \rightarrow g x, \quad g y_{n} \rightarrow g y_{1} \quad g z_{n} \rightarrow g z, \text { and } g w_{n} \rightarrow g w .
$$

Since $\left\{g x_{n}\right\},\left\{g z_{n}\right\}$ are non-decreasing and $\left\{g y_{n}\right\},\left\{g w_{n}\right\}$ are non-increasing, then since $(X, d, \leq)$ is regular we have

$$
g x_{n} \leq g x, g y_{n} \geq g y, g z_{n} \leq g z, g w_{n} \geq g w
$$

for all $n$. If $g x_{n}=g x, g y_{n}=g y, g z_{n}=g z$, and $g w_{n}=g w$ for some $n \geq 0$, then $g x=g x_{n} \leq$ $g x_{n+1} \leq g x=g x_{n}, g y \leq g y_{n+1} \leq g y_{n}=g y, g z=g z_{n} \leq g z_{n+1} \leq g z=g z_{n}$, and $g w \leq g w_{n+1} \leq$ $g w_{n}=g w$, which implies that

$$
g x_{n}=g x_{n+1}=F\left(x_{n}, y_{n}, z_{n}, w_{n}\right), \quad g y_{n}=g y_{n+1}=F\left(y_{n}, z_{n}, w_{n}, x_{n}\right),
$$

and

$$
g z_{n}=g z_{n+1}=F\left(z_{n}, w_{n}, x_{n}, y_{n}\right), \quad g w_{n}=g w_{n+1}=F\left(w_{n}, w_{n}, y_{n}, z_{n}\right),
$$

that is, $\left(x_{n}, y_{n}, z_{n}, w_{n}\right)$ is a quadruple coincidence point of $F$ and $g$. Then, we suppose that $\left(g x_{n}, g y_{n}, g z_{n}, g w_{n}\right) \neq(g x, g y, g z, g w)$ for all $n \geq 0$. By (3), consider now

$$
\begin{aligned}
& d(g x, F(x, y, z, w)) \leq d\left(g x, g x_{n+1}\right)+d\left(g x_{n+1}, F(x, y, z, w)\right) \\
& =d\left(g x, g x_{n+1}\right)+d\left(F\left(x_{n}, y_{n}, z_{n}, w_{n}\right), F(x, y, z, w)\right) \\
& \leq d\left(g x, g x_{n+1}\right)+\phi\left(\max \left\{\begin{array}{l}
d\left(g x_{n}, g x\right), d\left(g y_{n}, g y\right), \\
d\left(g z_{n}, g z\right), d\left(g w_{n}, g w\right)
\end{array}\right\}\right)+L M\left(x_{n}, y_{n}, z_{n}, w_{n}, x, y, z, w\right) \\
& <d\left(g x, g x_{n+1}\right)+\max \left\{d\left(g x_{n}, g x\right), d\left(g y_{n}, g y\right), d\left(g z_{n}, g z\right), d\left(g w_{n}, g w\right)\right\}+L M\left(x_{n}, y_{n}, z_{n}, w_{n}, x, y, z, w\right) .
\end{aligned}
$$

Taking $n \rightarrow \infty$ and using (44), the quantity $M\left(x_{n}, y_{n}, z_{n}, w_{n}, x, y, z, w\right)$ tends to 0 and so the right-hand side of (45) tends to 0 , hence we get that $d(g x, F(x, y, z, w))=0$. Thus, $g x=F(x, y, z, w)$. Analogously, one finds

$$
F(x, y, z, w)=g y, \quad F(z, w, x, y)=g z, \quad \text { and } \quad F(w, x, y, z)=g w .
$$

Thus, we proved that $F$ and $g$ have a quartet coincidence point. This completes the proof of Theorem 2.2.

Corollary 2.1 Let $(X, \leq)$ be a partially ordered set and suppose there is a metric $d$ on $X$ such that $(X, d)$ is a complete metric space. Suppose $F: X^{4} \rightarrow X$ and $g: X \rightarrow X$ are such that $F$ is continuous and has the mixed g-monotone property. Assume also that there exist $\varphi \in \Phi$ a non-decreasing function and $L \geq 0$ such that 


$$
\begin{aligned}
d(F(x, y, z, w), F(u, v, h, l)) & \leq \phi\left(\frac{d(g x, g u)+d(g y, g v)+d(g z, g h)+d(g w, g l}{4}\right) \\
& +L M(x, y, z, w, u, v, h, l),
\end{aligned}
$$

for any $x, y, z, w, u, v, h, l, \in X$ for which $g x \leq g u, g v \leq g y, g z \leq g w$, and $g l \leq g w$. Suppose $F\left(X^{4}\right) \subset g(X), g$ is continuous and commutes with $F$.

If there exist $x_{0}, y_{0}, z_{0}, w_{0} \in X$ such that $g x_{0} \leq F\left(x_{0}, y_{0}, z_{0}, w_{0}\right), g y_{0} \geq F\left(y_{0}, z_{0}, w_{0}\right.$, $\left.x_{0}\right), g z_{0} \leq F\left(z_{0}, w_{0}, x_{0}, y_{0}\right)$, and $g w_{0} \geq F\left(w_{0}, x_{0}, y_{0}, z_{0}\right)$, then there exist $x, y, z, w \in X$ such that

$$
F(x, y, z, w)=g x, \quad F(y, z, w, x)=g y, \quad F(z, w, x, y)=g z, \text { and } F(w, x, y, z)=g w .
$$

Proof. It suffices to remark that

$$
\frac{d(g x, g u)+d(g y, g v)+d(g z, p h), d(g w, g l)}{4} \leq \max \left\{\begin{array}{l}
d(g x, g u), d(g u, g v), \\
d(g z, g h), d(g w, g l)
\end{array}\right\} .
$$

Then, we apply Theorem 2.1, since $\varphi$ is assumed to be non-decreasing.

Similarly, as an easy consequence of Theorem 2.2 we have the following corollary.

Corollary 2.2 Let $(X, \leq)$ be a partially ordered set and suppose there is a metric $d$ on $X$ such that $(X, d, \leq)$ is regular. Suppose $F: X^{4} \rightarrow X$ and $g: X \rightarrow X$ are such that $F$ has the mixed g-monotone property. Assume also that there exist $\varphi \in \Phi$ a non-decreasing function and $L \geq 0$ such that

$$
\begin{aligned}
d(F(x, y, z, w), F(u, v, h, l)) & \leq \phi\left(\frac{d(g x, g u)+d(g y, g v)+d(g z, g h)+d(g w, g l)}{4}\right) \\
& +\operatorname{LM}(x, y, z, w, u, v, h, l),
\end{aligned}
$$

for any $x, y, z, w, u, v, h, l \in X$ for which $g x \leq g u, g v \leq g y, g z \leq g w$, and $g l \leq g w$. Also, suppose $F\left(X^{4}\right) \subset g(X)$ and $(g(X), d)$ is a complete metric space.

If there exist $x_{0}, y_{0}, z_{0}, w_{0} \in X$ such that $g x_{0} \leq F\left(x_{0}, y_{0}, z_{0}, w_{0}\right), g y_{0} \geq F\left(y_{0}, z_{0}, w_{0}\right.$, $\left.x_{0}\right), g z_{0} \leq F\left(z_{0}, w_{0}, x_{0}, y_{0}\right)$, and $g w_{0} \geq F\left(w_{0}, x_{0}, y_{0}, z_{0}\right)$, then there exist $x, y, z, w \in X$ such that

$$
F(x, y, z, w)=g x, \quad F(y, z, w, x)=g y, \quad F(z, w, x, y)=g z, \quad \text { and } \quad F(w, x, y, z)=g w .
$$

Corollary 2.3 Let $(X, \leq)$ be a partially ordered set and suppose there is a metric $d$ on $X$ such that $(X, d)$ is a complete metric space. Suppose $F: X^{4} \rightarrow X$ and $g: X \rightarrow X$ are such that $F$ is continuous and has the mixed g-monotone property. Assume that there exist $k \in[0,1)$ and $L \geq 0$ such that

$$
d(F(x, y, z, w), F(u, v, h, l)) \leq k \max \left\{\begin{array}{l}
d(g x, g u), d(g y, g v), \\
d(g z, g h), d(g w, g l)
\end{array}\right\}+L M(x, y, z, w, u, v, h, l),
$$

for any $x, y, z, w, u, v, h, l \in X$ for which: $g x \leq g u, g v \leq g y, g z \leq g w$, and $g l \leq g w$. Suppose $F\left(X^{4}\right) \subset g(X), g$ is continuous and commutes with $F$.

If there exist $x_{0}, y_{0}, z_{0}, w_{0} \in X$ such that $g x_{0} \leq F\left(x_{0}, y_{0}, z_{0}, w_{0}\right), g y_{0} \geq F\left(y_{0}, z_{0}, w_{0}\right.$, $\left.x_{0}\right), g z_{0} \leq F\left(z_{0}, w_{0}, x_{0}, y_{0}\right)$, and $g w_{0} \geq F\left(w_{0}, x_{0}, y_{0}, z_{0}\right)$, then there exist $x, y, z, w \in X$ such that 


$$
F(x, y, z, w)=g x, \quad f(y, z, w, x)=g y, \quad f(z, w, x, y)=g z, \text { and } F(w, x, y, z)=g w .
$$

Proof. It suffices to take $\varphi(t)=k t$ in Theorem 2.1.

Corollary 2.4 Let $(X, \leq)$ be a partially ordered set and suppose there is a metric $d$ on $X$ such that $(X, d, \leq)$ is regular. Suppose $F: X^{4} \rightarrow X$ and $g: X \rightarrow X$ are such that $F$ has the mixed g-monotone property. Assume that there exist $k \in[0,1)$ and $L \geq 0$ such that

$$
d(F(x, y, z, w), F(u, v,, h, l)) \leq k \max \left\{\begin{array}{l}
d(g x, g u), d(g y, g v), \\
d(g z, g h), d(g w, g l)
\end{array}\right\}+L M(x, y, z, w, u, v, h, l),
$$

for any $x, y, z, w, u, v, h, l \in X$ for which $g x \leq g u, g v \leq g y, g z \leq g w$, and $g l \leq g w$. Suppose $F\left(X^{4}\right) \subset g(X)$ and $(g(X), d)$ is a complete metric space.

If there exist $x_{0}, y_{0}, z_{0}, w_{0} \in X$ such that $g x_{0} \leq F\left(x_{0}, y_{0}, z_{0}, w_{0}\right), g y_{0} \geq F\left(y_{0}, z_{0}, w_{0}, x_{0}\right)$, $g z_{0} \leq F\left(z_{0}, w_{0}, x_{0}, y_{0}\right)$, and $g w_{0} \geq F\left(w_{0}, x_{0}, y_{0}, z_{0}\right)$, then there exist $x, y, z, w \in X$ such that

$$
F(x, y, z, w)=g x, \quad F(y, z, w, x)=g \gamma, \quad F(z, w, x, y)=g z, \quad \text { and } \quad F(w, x, y, z)=g w .
$$

Proof. It suffices to take $\varphi(t)=k t$ in Theorem 2.2.

Corollary 2.5 Let $(X, \leq)$ be a partially ordered set and suppose there is a metric $d$ on $X$ such that $(X, d)$ is a complete metric space. Suppose $F: X^{4} \rightarrow X$ and $g: X \rightarrow X$ are such that $F$ is continuous and has the mixed g-monotone property. Assume that there exist $k \in[0,1)$ and $L \geq 0$ such that

$$
d(F(x, y, z, w), F(u, v, h, l)) \leq \frac{k}{4}\left\{\begin{array}{l}
d(g x, g u)+d(g y, g v)+ \\
d(g z, g h)+d(g w, g l)
\end{array}\right\}+L M(x, y, z, w, u, v, h, l),
$$

for any $x, y, z, w, \in X$ for which $: g x \leq g u, g v \leq g y, g z \leq g w$, and $g l \leq g w$. Also, suppose $F\left(X^{4}\right) \subset g(X)$ and $(g(X), g$ is continuous and commutes with $F$.

If there exist $x_{0}, y_{0}, z_{0}, w_{0} \in X$ such that $g x_{0} \leq F\left(x_{0}, y_{0}, z_{0}, w_{0}\right), g y_{0} \geq F\left(y_{0}, z_{0}, w_{0}, x_{0}\right)$, $g z_{0} \leq F\left(z_{0}, w_{0}, x_{0}, y_{0}\right)$, and $g w_{0} \geq F\left(w_{0}, x_{0}, y_{0}, z_{0}\right)$, then there exist $x, y, z, w \in X$ such that

$$
F(x, y, z, w)=g x, F(y, z, w, x)=g y, F(z, w, x, y)=g z, \text { and } F(w, x, y, z)=g w .
$$

Proof. It suffices to take $\varphi(t)=k t$ in Corollary 2.1.

Corollary 2.6 Let $(X, \leq)$ be a partially ordered set and suppose there is a metric $d$ on $X$ such that $(X, d, \leq)$ is regular. Suppose $F: X^{4} \rightarrow X$ and $g: X \rightarrow X$ are such that $F$ has the mixed g-monotone property. Assume that there exist $k \in[0,1)$ and $L \geq 0$ such that

$$
d(F(x, y, z, w), F(u, v, h, l)) \leq \frac{k}{4}\left\{\begin{array}{l}
d(g x, g u)+d(g y, g v)+ \\
d(g z, g h)+d(g w, g l)
\end{array}\right\}+L M(x, y, z, w, u, v, h, l),
$$

for any $x, y, z, w, \in X$ for which $g x \leq g u, g v \leq g y, g z \leq g w$, and $g l \leq g w$. Suppose $F\left(X^{4}\right)$ $\subset g(X)$ and $(g(X), d)$ is a complete metric space.

If there exist $x_{0}, y_{0}, z_{0}, w_{0} \in X$ such that $g x_{0} \leq F\left(x_{0}, y_{0}, z_{0}, w_{0}\right), g y_{0} \geq F\left(y_{0}, z_{0}, w_{0}, x_{0}\right)$, $g z_{0} \leq F\left(z_{0}, w_{0}, x_{0}, y_{0}\right)$, and $g w_{0} \geq F\left(w_{0}, x_{0}, y_{0}, z_{0}\right)$, then there exist $x, y, z, w \in X$ such that 


$$
F(x, y, z, w)=g x, \quad F(y, z, w, r)=g y, \quad F(z, w, x, y)=g z, \quad \text { and } F(w, x, y, z=g w)
$$

Proof. It suffices to take $\varphi(t)=k t$ in Corollary 2.2.

Remark 1 - Corollary 2.4 of Karapinar [39] is a particular case of Corollary 2.5 by taking $L=0$ and $g=I_{X}$ the identity on $X$.

- Corollary 2.4 of Karapinar [39]is a particular case of Corollary 2.6 by taking L = 0 and $g=I_{X}$.

- Theorem 2.6 of Berinde and Karapinar [40] is a particular case of Corollary 2.1 by taking $L=0$.

- Theorem 2.6 of Berinde and Karapinar [40] is a particular case of Corollary 2.1 by taking $L=0$.

Now, we shall prove the existence and uniqueness of quadruple common fixed point. For a product $X^{4}$ of a partial ordered set $(X, \leq)$, we define a partial ordering in the following way: For all $(x, y, z, w),(u, v, r, h) \in X^{4}$

$$
(x, y, z, w) \leq(u, v, r, h) \Leftrightarrow x \leq u, \quad y \geq v, \quad z \leq r \text { and } w \geq l
$$

We say that $(x, y, z, w)$ and $(u, v, r, l)$ are comparable if

$$
(x, y, z, w) \leq(u, v, r, l) \quad \text { or } \quad(u, v, r, l) \leq(x, y, z, w) .
$$

Also, we say that $(x, y, z, w)$ is equal to $(u, v, r, l)$ if and only if $x=u, y=v, z=r$ and $w=l$.

Theorem 2.3 In addition to hypotheses of Theorem 2.1, suppose that for all (x, y, $z$, $w),(u, v, r, l) \in X^{4}$, there exists $(a, b, c, d) \in X^{4}$ such that

$$
(F(a, b, c, d), F(b, c, d, a), F(c, d, a, b), F(d, a, b, c))
$$

is comparable to

$$
\begin{gathered}
(F(x, y, z, w), F(y, z, w, x), F(z, w, x, y), F(w, x, y, z)) \text { and } \\
(F(u, v, r, l), F(v, r, l, u), F(r, l, u, v), F(l, u, v, r)) .
\end{gathered}
$$

Then, $F$ and $g$ have a unique quadruple common fixed point $(x, y, z, w)$ such that

$$
\begin{aligned}
x & =g x=F(x, y, z, w), \\
z & =g z=F(z, w, x, y), \quad \text { and } \quad \begin{aligned}
y & =g y=F(y, z, w, x),
\end{aligned}=g w=F(w, x, y, z) .
\end{aligned}
$$

Proof. The set of quadruple coincidence points of $F$ and $g$ is not empty due to Theorem 2.1. Assume, now, $(x, y, z, w)$ and $(u, v, r, l)$ are two quadruple coincidence points of $F$ and $g$, that is,

$$
\begin{array}{ll}
F(x, y, z, w)=g x, & F(u, v, r, l)=g u, \\
F(y, z, w, x)=g y, & F(v, r, l, u)=g v, \\
F(z, w, x, y)=g z, & F(r, l, u, v)=g r, \\
F(w, x, y, z)=g w, & F(l, u, v, r)=g l .
\end{array}
$$

We shall show that $(g x, g y, g z, g w)$ and $(g u, g v, g r, g l)$ are equal. By assumption, there exists $(a, b, c, d) \in X^{4}$ such that $(F(a, b, c, d), F(b, c, d, a), F(c, d, a, b), F(d, a, b, c))$ 
is comparable to $(F(x, y, z, w), F(y, z, w, x), F(z, w, x, y), F(w, x, y, z))$ and $(F(u, v, r$, $l), F(v, r, l, u), F(r, l, u, v), F(l, u, v, r))$.

Define sequences $\left\{g a_{n}\right\},\left\{g b_{n}\right\}$, $\left\{g c_{n}\right\}$, and $\left\{g d_{n}\right\}$ such that

$$
\begin{aligned}
a_{0} & =a, b_{0}=b, c_{0}=\mathrm{c}, d_{0}=\mathrm{d} \text { and for any } n \geq 1 \\
g a_{n} & =F\left(a_{n-1}, b_{n-1}, c_{n-1}, d_{n-1}\right), \\
g b_{n} & =F\left(b_{n-1}, c_{n-1}, d_{n-1}, a_{n-1}\right), \\
g c_{n} & =F\left(c_{n-1}, d_{n-1}, a_{n-1}, b_{n-1}\right), \\
g d_{n} & =F\left(d_{n-1}, a_{n-1}, b_{n-1}, c_{n-1}\right),
\end{aligned}
$$

for all $n$. Further, set $x_{0}=x, y_{0}=y, z_{0}=z, w_{0}=w$ and $u_{0}=u, v_{0}=v, r_{0}=r, l_{0}=l$ and on the same way define the sequences $\left\{g x_{n}\right\},\left\{g y_{n}\right\},\left\{g z_{n}\right\},\left\{g w_{n}\right\}$ and $\left\{g u_{n}\right\},\left\{g v_{n}\right\}$, $\left\{g r_{n}\right\},\left\{g l_{n}\right\}$. Then, it is easy that

$$
\begin{array}{ll}
g x_{n}=F(x, y, z, w), & g u_{n}=F(u, v, r, l), \\
g y_{n}=F(y, z, w, x), & g v_{n}=F(v, r, l, u), \\
g z_{n}=F(z, w, x, y), & g r_{n}=F(r, l, u, v), \\
g w_{n}=F(w, x, y, z), & g l_{n}=F(l, u, v, r)
\end{array}
$$

for all $n \geq 1$. Since

$$
\begin{aligned}
(F(x, y, z, w), F(y, z, w, x), F(z, w, x, y), F(w, x, y, z)) & =\left(g x_{1}, g y_{1}, g z_{1}, g w_{1}\right) \\
& =(g x, g y, g z, g w)
\end{aligned}
$$

is comparable to

$$
(F(a, b, c, d), F(b, c, d, a), F(c, d, a, b), F(d, a, b, c))=\left(g a_{1}, g b_{1}, g c_{1}, g d_{1}\right),
$$

then it is easy to show $(g x, g y, g z, g w) \geq\left(g a_{1}, g b_{1}, g c_{1}, g d_{1}\right)$. Recursively, we get that

$$
\left(g a_{n}, g b_{n}, g c_{n}, g d_{n}\right) \leq(g x, g y, g z, g w) \text { for all } n \text {. }
$$

From (2) and (47), it is obvious that

$$
\begin{aligned}
& \left.M\left(a_{n}, b_{n}, c_{n}, d_{n}, x, y, z, w\right)=M\left(y, z, w, x, b_{n}, c_{n}, d_{n}, a_{n}\right)\right) \\
= & M\left(c_{n}, d_{n}, a_{n}, b_{n}, z, w, x, y\right)=M\left(w, x, y, z, d_{n}, a_{n}, b_{n}, c_{n}\right)=0 .
\end{aligned}
$$

By (50), (51), and (3), we have

$$
\begin{aligned}
& d\left(g a_{n+1}, g x\right)=d\left(F\left(a_{n}, b_{n}, c_{n}, d_{n}\right), F(x, y, z, w)\right) \\
& \leq \phi\left(\max \left\{d\left(g x, g a_{n}\right), d\left(g y, g b_{n}\right), d\left(g z, g c_{n}\right), d\left(g w, g d_{n}\right)\right\}\right), \\
& \quad+L M\left(a_{n}, b_{n}, c_{n}, d_{n}, x, y, z, w\right) \\
& =\phi\left(\max \left\{d\left(g x, g a_{n}\right), d\left(g y, g b_{n}\right), d\left(g z, g c_{n}\right), d\left(g w, g d_{n}\right)\right\}\right), \\
& d\left(g y, g b_{n+1}\right)=d\left(F(y, z, w, x), F\left(b_{n}, c_{n}, d_{n}, a_{n}\right)\right) \\
& \leq \phi\left(\max \left\{d\left(g a_{n}, g x\right), d\left(g b_{n}, g y\right), d\left(g c_{n}, g z\right), d\left(g d_{n}, g w\right)\right\}\right) \\
& \quad+L M\left(y, z, w, x, b_{n}, c_{n}, d_{n}, a_{n}\right) \\
& =\phi\left(\max \left\{d\left(g a_{n}, g x\right), d\left(g b_{n}, g y\right), d\left(g c_{n}, g z\right), d\left(g d_{n}, g w\right)\right\}\right)
\end{aligned}
$$




$$
\begin{aligned}
& d\left(g c_{n+1}, g z\right)=d\left(F\left(c_{n}, d_{n}, a_{n}, b_{n}\right), F(z, w, x, y)\right) \\
& \leq \phi\left(\max \left\{d\left(g a_{n}, g x\right), d\left(g b_{n}, g y\right), d\left(g c_{n}, g z\right), d\left(g d_{n}, g w\right)\right\}\right) \\
& \quad+L M\left(c_{n}, d_{n}, a_{n}, b_{n}, z, w, x, y\right) \\
& =\phi\left(\max \left\{d\left(g a_{n}, g x\right), d\left(g b_{n}, g y\right), d\left(g c_{n}, g z\right), d\left(g d_{n}, g w\right)\right\}\right)
\end{aligned}
$$

and

$$
\begin{aligned}
& d\left(g w, g d_{n+1}\right)=d\left(F(w, x, y, z), F\left(d_{n}, a_{n}, b_{n}, c_{n}\right)\right) \\
& \leq \phi\left(\max \left\{d\left(g a_{n}, g x\right), d\left(g b_{n}, g y\right), d\left(g c_{n}, g z\right), d\left(g d_{n}, g w\right)\right\}\right) \\
& \quad+L M\left(w, x, y, z, d_{n}, a_{n}, b_{n}, c_{n}\right) \\
& =\phi\left(\max \left\{d\left(g d_{n}, g w\right), d\left(g a_{n}, g x\right), d\left(g b_{n}, g y\right), d\left(g c_{n}, g z\right)\right\}\right) .
\end{aligned}
$$

From (52)-(55), it follows that

$$
\max \left\{\begin{array}{l}
d\left(g z, g c_{n+1}\right), d\left(g y, g b_{n+1}\right), \\
d\left(g x, g a_{n+1}\right), d\left(g w, g d_{n+1}\right)
\end{array}\right\} \leq \phi\left(\max \left\{\begin{array}{l}
d\left(g z, g c_{n}\right), d\left(g y, g b_{n}\right), \\
d\left(g x, g a_{n}\right), d\left(g w, g d_{n}\right)
\end{array}\right\}\right) .
$$

Therefore, for each $n \geq 1$,

$$
\max \left\{\begin{array}{l}
d\left(g z, g c_{n}\right), d\left(g y, g b_{n}\right), \\
d\left(g x, g a_{n}\right), d\left(g w, g d_{n}\right)
\end{array}\right\} \leq \phi^{n}\left(\max \left\{\begin{array}{l}
d\left(g z, g c_{0}\right), d\left(g y, g b_{0}\right), \\
d\left(g x, g a_{0}\right), d\left(g w, g d_{0}\right)
\end{array}\right\}\right) .
$$

It is known that $\varphi(t)<t$ and $\lim _{r \rightarrow t^{+}} \phi(r)<t$ imply $\lim _{n \rightarrow \infty} \phi^{n}(t)=0$ for each $t>0$. Thus, from (57)

$$
\lim _{n \rightarrow \infty} \max \left\{d\left(g z, g c_{n}\right), d\left(g y, g b_{n}\right), d\left(g x, g a_{n}\right), d\left(g w, g d_{n}\right)\right\}=0 .
$$

This yields that

$$
\lim _{n \rightarrow \infty} d\left(g x, g a_{n}\right)=0, \quad \lim _{n \rightarrow \infty} d\left(g y, g b_{n}\right)=0, \lim _{n \rightarrow \infty} d\left(g z, g c_{n}\right)=0 \text { and } \lim _{n \rightarrow \infty} d\left(g w, g d_{n}\right)=0 .
$$

Analogously, we may show that

$$
\lim _{n \rightarrow \infty} d\left(g u, g a_{n}\right)=0, \lim _{n \rightarrow \infty} d\left(g v, g b_{n}\right)=0, \lim _{n \rightarrow \infty} d\left(g r, g c_{n}\right)=0 \text { and } \lim _{n \rightarrow \infty} d\left(g l, g d_{n}\right)=0 .
$$

Combining (58) and (59) yields that $(g x, g y, g z, g w)$ and ( $g u, g v, g r, g l)$ are equal.

Since $g x=F(x, y, z, w), g y=F(y, z, w, x), g z=F(z, w, x . y)$, and $g z=F(z, w, x, y)$, by commutativity of $F$ and $g$ we have

$$
\begin{aligned}
& g x^{\prime}=g(g x)=g(F(x, y, z, w))=F(g x, g y, g z, g w), \\
& g y^{\prime}=g(g y)=g(F(y, z, w, x))=F(g y, g z, g w, g x), \\
& g z^{\prime}=g(g z)=g(F(z, w, x, y))=F(g z, g w, g x, g y)
\end{aligned}
$$

and

$$
g w^{\prime}=g(g w)=g(F(w, x, y, z))=F(g w, g x, g y, g z)
$$

where $g x=x^{\prime}, g y=y^{\prime}, g z=z^{\prime}$, and $g w=w^{\prime}$. Thus, $\left(x^{\prime}, y^{\prime}, z^{\prime}, w^{\prime}\right)$ is a quadruple coincidence point of $F$ and $g$. Consequently, $\left(g x^{\prime}, g y^{\prime}, g z^{\prime}, g z^{\prime}\right)$ and $(g x, g y, g z, g w)$ are equal. We deduce

$$
g x^{\prime}=g x=x^{\prime}, \quad g y^{\prime}=g y=y^{\prime} \text { and } g z^{\prime}=g z=z^{\prime}, \quad g w^{\prime}=g w=w^{\prime} .
$$


Therefore, $\left(x^{\prime}, y^{\prime}, z^{\prime}, w^{\prime}\right)$ is a quadruple common fixed of $F$ and $g$. Its uniqueness follows easily from (3).

Example 2.1 Let $X=\mathbb{R}$ be endowed with the usual ordering and the usual metric, which is complete.

Let $g: X \rightarrow X$ and $F: X^{4} \rightarrow X$ be defined by

$$
g(x)=\frac{3}{4} x, \quad F(x, y, z, w)=\frac{x-y+z-w}{8}, \text { for all } x, y, z, w \in X
$$

Take $\varphi:[0, \infty) \rightarrow[0, \infty)$ be given by $\phi(t)=\frac{2}{3} t$ for all $t \in[0, \infty)$.

We will check that the contraction (3) is satisfied for all $x, y, z, w, u, v, h, l \in X$ satisfying $g x \leq g u, g v \leq g y, g z \leq g h$, and $g l \leq g w$. In this case, we have

$$
\begin{aligned}
d(F(x, y, z, w), F(u, v, h, l))= & \frac{u-x}{8}+\frac{y-v}{8}+\frac{h-z}{8}+\frac{w-l}{8} \\
\leq & \frac{1}{2}[\max \{(u-x),(y-v),(h-z),(w-l)\}] \\
= & \frac{2}{3} \max \{d(g x, g u), d(g y, g v), d(g z, g h), d(g w, g l)\} \\
\leq & \phi(\max \{d(g x, g u), d(g y, g v), d(g z, g h), d(g w, g l)\}) \\
& +L M(x, y, z, w, u, v, h, l),
\end{aligned}
$$

for arbitrary $L \geq 0$.

It is obvious that the other hypotheses of Theorem 2.3 are satisfied. We deduce that $(0,0,0,0)$ is the unique quadruple common fixed point of $F$ and $g$.

\section{Application to matrix equations}

In this section, we study the existence and uniqueness of solutions $(X, Y, Z, T)$ to the system of matrix equations

$$
\left\{\begin{array}{l}
X=Q+A_{1}^{*} X A_{1}-B_{1}^{*} Y B_{1}+A_{2}^{*} Z A_{2}-B_{2}^{*} T B_{2} \\
Y=Q+A_{1}^{*} Y A_{1}-B_{1}^{*} Z B_{1}+A_{2}^{*} T A_{2}-B_{2}^{*} X B_{2} \\
Z=Q+A_{1}^{*} Z A_{1}-B_{1}^{*} T B_{1}+A_{2}^{*} X A_{2}-B_{2}^{*} Y B_{2} \\
T=Q+A_{1}^{*} T A_{1}-B_{1}^{*} X B_{1}+A_{2}^{*} Y A_{2}-B_{2}^{*} Z B_{2}
\end{array}\right.
$$

where $A_{1}, A_{2}, B_{1}, B_{2} \in \mathcal{M}(n)$ : the set of all $n \times n$ matrices, $Q \in \mathcal{P}(n)$ : the set of all $n \times n$ positive definite matrices, and $\mathcal{H}(n)$ is the set of all $n \times n$ Hermitian matrices.

We endow $\mathcal{H}(n)$ with the partial order $\leqslant$ given by

$$
M, N \in \mathcal{H}(n), \quad M \preccurlyeq N \Leftrightarrow N-M \in \mathcal{P}(n) .
$$

For a fixed $P \in \mathcal{P}(n)$, we consider

$$
\|H\|_{1, P}=\operatorname{tr}\left(P^{\frac{1}{2}} H P^{\frac{1}{2}}\right) .
$$

for all $H \in \mathcal{H}(n)$, where $t r$ is the trace operator. The space $\mathcal{H}(n)$ equipped with the metric induced by $\|.\|_{1, P}$ is a complete metric space for any positive definite matrix $P$ (see [42]).

The following lemma will be useful for our application. 
Lemma 3.1 Let $A \geqslant 0$ and $B \geqslant 0$ be $n \times n$ matrices. Then, we have

$$
0 \leq \operatorname{tr}(A B)=\operatorname{tr}(B A) \leq\|A\| \operatorname{tr}(B),
$$

where $\|$.$\| is the spectral norm.$

Theorem 3.1 Suppose that there exists $P \in \mathcal{P}(n)$ such that

$$
k=4 \max \left\{\left\|P^{-\frac{1}{2}} A_{1}^{*} P A_{1} P^{-\frac{1}{2}}\right\|,\left\|P^{-\frac{1}{2}} A_{2}^{*} P A_{2} P^{-\frac{1}{2}}\right\|,\left\|P^{-\frac{1}{2}} B_{1}^{*} P B_{1} P^{-\frac{1}{2}}\right\|,\left\|P^{-\frac{1}{2}} B_{2}^{*} P B_{2} P^{-\frac{1}{2}}\right\|\right\}<1 .
$$

Suppose also that

$$
0 \preccurlyeq \sum_{i=1}^{2} A_{i}^{*} Q A_{i} \text { and } Q \preccurlyeq \sum_{i=1}^{2} B_{i}^{*} Q B_{i} .
$$

Then, the system (60) has one and only one solution $\left(X_{1}, X_{2}, X_{3}, X_{4}\right) \in(\mathcal{H}(n))^{4}$.

Proof. Consider the mappings $F:(\mathcal{H}(n))^{4} \rightarrow \mathcal{H}(n)$ and $g: \mathcal{H}(n) \rightarrow \mathcal{H}(n)$ defined by

$$
F\left(X_{1}, X_{2}, X_{3}, X_{4}\right)=Q+A_{1}^{*} X_{1} A_{1}-B_{1}^{*} X_{2} B_{1}+A_{2}^{*} X_{3} A_{2}-B_{2}^{*} X_{4} B_{2} \text { and } g X=X \text {, }
$$

for all $X, X_{i} \in \mathcal{H}(n) i=1, \ldots, 4$.

For all $X_{i}, Y_{i} \in \mathcal{H}(n) i=1 \ldots, 4$ with $g X_{1} \leqslant g Y_{1}, g Y_{2} \leqslant g X_{2}, g X_{3} \leqslant g Y_{3}$ and $g Y_{4} \preccurlyeq g X_{4}$, by using Lemma 3.1, we have

$$
\begin{aligned}
& \left\|F\left(Y_{1}, Y_{2}, Y_{3}, Y_{4}\right)-F\left(X_{1}, X_{2}, X_{3}, X_{4}\right)\right\|_{1, P} \\
& =\left\|A_{1}^{*}\left(Y_{1}-X_{1}\right) A_{1}-B_{1}^{*}\left(Y_{2}-X_{2}\right) B_{1}+A_{2}^{*}\left(Y_{3}-X_{3}\right) A_{2}-B_{2}^{*}\left(Y_{4}-X_{4}\right) B_{2}\right\|_{1, P} \\
& =\operatorname{tr}\left[P^{\frac{1}{2}}\left(A_{1}^{*}\left(Y_{1}-X_{1}\right) A_{1}-B_{1}^{*}\left(Y_{2}-X_{2}\right) B_{1}+A_{2}^{*}\left(Y_{3}-X_{3}\right) A_{2}-B_{2}^{*}\left(Y_{4}-X_{4}\right) B_{2}\right) P^{\frac{1}{2}}\right] \\
& =\operatorname{tr}\left[A_{1} P A_{1}^{*}\left(Y_{1}-X_{1}\right)\right]+\operatorname{tr}\left[B_{1} P B_{1}^{*}\left(X_{2}-Y_{2}\right)\right]+\operatorname{tr}\left[A_{2} P A_{2}^{*}\left(Y_{3}-X_{3}\right)\right]+\operatorname{tr}\left[B_{2} P B_{2}^{*}\left(X_{4}-Y_{4}\right)\right] \\
& =\operatorname{tr}\left[A_{1} P A_{1}^{*} P^{-\frac{1}{2}} P^{\frac{1}{2}}\left(Y_{1}-X_{1}\right) P^{\frac{1}{2}} P^{-\frac{1}{2}}\right]+\operatorname{tr}\left[B_{1} P B_{1}^{*} P^{-\frac{1}{2}} P^{\frac{1}{2}}\left(X_{2}-Y_{2}\right) P^{\frac{1}{2}} P^{-\frac{1}{2}}\right] \\
& +\operatorname{tr}\left[A_{2} P A_{2}^{*} P^{-\frac{1}{2}} P^{\frac{1}{2}}\left(Y_{3}-X_{3}\right) P^{\frac{1}{2}} P^{-\frac{1}{2}}\right]+\operatorname{tr}\left[B_{2} P B_{2}^{*} P^{-\frac{1}{2}} P^{\frac{1}{2}}\left(X_{4}-Y_{4}\right) P^{\frac{1}{2}} P^{-\frac{1}{2}}\right] \\
& \leq\left\|P^{-\frac{1}{2}} A_{1} P A_{1}^{*} P^{-\frac{1}{2}}\right\| \operatorname{tr}\left(P^{\frac{1}{2}}\left(Y_{1}-X_{1}\right) P^{\frac{1}{2}}\right)+\left\|P^{-\frac{1}{2}} B_{1} P B_{1}^{*} P^{-\frac{1}{2}}\right\| \operatorname{tr}\left(P^{\frac{1}{2}}\left(X_{2}-Y_{2}\right) P^{\frac{1}{2}}\right) \\
& +\left\|P^{-\frac{1}{2}} A_{2} P A_{2}^{*} P^{-\frac{1}{2}}\right\| \operatorname{tr}\left(P^{\frac{1}{2}}\left(Y_{3}-X_{3}\right) P^{\frac{1}{2}}\right)+\left\|P^{-\frac{1}{2}} B_{2} P B_{2}^{*} P^{-\frac{1}{2}}\right\| \operatorname{tr}\left(P^{\frac{1}{2}}\left(X_{4}-Y_{4}\right) P^{\frac{1}{2}}\right) \\
& =\left\|P^{-\frac{1}{2}} A_{1} P A_{1}^{*} P^{-\frac{1}{2}}\right\|\left\|Y_{1}-X_{1}\right\|_{1, P}+\left\|P^{-\frac{1}{2}} B_{1} P B_{1}^{*} P^{-\frac{1}{2}}\right\|\left\|X_{2}-Y_{2}\right\|_{1, P} \\
& +\left\|P^{-\frac{1}{2}} A_{2} P A_{2}^{*} P^{-\frac{1}{2}}\right\|\left\|Y_{3}-X_{3}\right\|_{1, P}+\left\|P^{-\frac{1}{2}} B_{2} P B_{2}^{*} P^{-\frac{1}{2}}\right\|\left\|X_{4}-Y_{4}\right\|_{1, P} \\
& \leq \frac{k}{4}\left(\left\|g Y_{1}-g X_{1}\right\|_{1, P}+\left\|g X_{2}-g Y_{2}\right\|_{1, P}+\left\|g Y_{3}-g X_{3}\right\|_{1, P}+\left\|g X_{4}-g Y_{4}\right\|_{1, P}\right) .
\end{aligned}
$$

Thus, we proved that the contractive condition given in Corollary 2.5 is satisfied for all $L \geq 0$. Moreover, from (62), we have letting $g Q \preccurlyeq F(Q, 0, Q, 0)$ and $g 0 \geqslant F(0, Q, 0$, $Q$ ). Applying Corollary 2.5, $F$ and $g$ have a coupled coincidence point (and so a quadrupled fixed point since $g$ is the identity on $\mathcal{H}(n))$. Then, there exist $X_{1}, X_{2}, X_{3}, X_{4} \in \mathcal{H}(n)$ such that

$$
\begin{gathered}
F\left(X_{1}, X_{2}, X_{3}, X_{4}\right)=X_{1}, \quad F\left(X_{2}, X_{3}, X_{4}, X_{1}\right)=X_{2} \\
F\left(X_{3}, X_{4}, X_{1}, X_{2}\right)=X_{3} \quad \text { and } \quad F\left(X_{4}, X_{1}, X_{2}, X_{4}\right)=X_{4} .
\end{gathered}
$$

On the other hand, for all $X, Y \in \mathcal{H}(n)$ there is a greatest lower bound and a least upper bound, hence it is obvious that the hypotheses of Theorem 2.3 hold, so the 
uniqueness of that quadrupled fixed point of $F$, which is also the unique solution of the system (60).

\section{Author details}

${ }^{1}$ Department of Mathematics, The Hashemite University, P.O. 330127, Zarqa 13115, Jordan ${ }^{2}$ Institut Supérieur d'Informatique et des Technologies de Communication de Hammam Sousse, Université de Sousse, Route GP1-4011, Hammam Sousse, Tunisie ${ }^{3}$ Department of Mathematics, Atilim University, 06836, İncek, Ankara, Turkey

\section{Authors' contributions}

All authors have contributed in obtaining the new results presented in this article. All authors read and approve the final manuscript.

\section{Competing interests}

The authors declare that they have no competing interests.

\section{Received: 19 December 2011 Accepted: 1 May 2012 Published: 1 May 2012}

\section{References}

1. Turinici, M: Abstract comparison principles and multivariable Gronwall-Bellman in-equalities. J Math Anal Appl. 117, 100-127 (1986). doi:10.1016/0022-247X(86)90251-9

2. Ran, ACM, Reurings, MCB: A fixed point theorem in partially ordered sets and some application to matrix equations. Proc Am Math Soc. 132, 1435-1443 (2004). doi:10.1090/50002-9939-03-07220-4

3. Abbas, M, Sintunavarat, W, Kumam, P: Coupled fixed point in partially ordered G-metric spaces. Fixed Point Theory Appl. 2012, 31 (2012). doi:10.1186/1687-1812-2012-31

4. Altun, I, Simsek, H: Some fixed point theorems on ordered metric spaces and application. Fixed Point Theory Appl 2010 (2010). Article ID 621492

5. Aydi, $\mathrm{H}$ : Coincidence and common fixed point results for contraction type maps in partially ordered metric spaces. Int J Math Anal. 5(13):631-642 (2011)

6. Choudhury, BS, Metiya, N, Kundu, A: Coupled coincidence point theorems in ordered metric spaces. Ann Univ Ferrara. 57, 1-16 (2011). doi:10.1007/s11565-011-0117-5

7. Choudhury, BS, Kundu, A: A coupled coincidence point result in partially ordered metric spaces for compatible mappings. Nonlinear Anal. 73, 2524-2531 (2010). doi:10.1016/j.na.2010.06.025

8. Harjani, J, Sadarangani, K: Fixed point theorems for weakly contractive mappings in partially ordered sets. Nonlinear Anal. 71, 3403-3410 (2009). doi:10.1016/j.na.2009.01.240

9. Luong, NV, Thuan, NX: Coupled fixed points in partially ordered metric spaces and application. Nonlinear Anal. 74, 983-992 (2011). doi:10.1016/j.na.2010.09.055

10. Nashine, HK, Samet, B: Fixed point results for mappings satisfying $(\psi, \bigotimes)$-weakly contractive condition in partially ordered metric spaces. Nonlinear Anal. 74, $2201-2209$ (2011). doi:10.1016/j.na.2010.11.024

11. Nashine, HK, Shatanawi, W: Coupled common fixed point theorems for a pair of commuting mappings in partially ordered complete metric spaces. Comput Math Appl. 62, 1984-1993 (2011). doi:10.1016/j.camwa.2011.06.042

12. Nieto, JJ, López, RR: Contractive mapping theorems in partially ordered sets and applications to ordinary differential equations. Order. 22, 223-239 (2005). doi:10.1007/s11083-005-9018-5

13. Samet, B: Coupled fixed point theorems for a generalized Meir-Keeler contraction in partially ordered metric spaces Nonlinear Anal. 7(12):4508-4517 (2010)

14. Bhaskar, TG, Lakshmikantham, V: Fixed point theory in partially ordered metric spaces and applications. Nonlinear Anal. 65, 1379-1393 (2006). doi:10.1016/j.na.2005.10.017

15. Lakshmikantham, V, Ćirić, LjB: Coupled fixed point theorems for nonlinear contractions in partially ordered metric spaces. Nonlinear Anal. 70, 4341-4349 (2009). doi:10.1016/j.na.2008.09.020

16. Abbas, M, Khan, MA, Radenović, S: Common coupled fixed point theorem in cone metric space for $w$-compatible mappings. Appl Math Comput. 217, 195-202 (2010). doi:10.1016/j.amc.2010.05.042

17. Aydi, H: Some coupled fixed point results on partial metric spaces. Int J Math Math Sci 2011 (2011). Article ID 647091, 11

18. Aydi, H, Samet, B, Vetro, C: Coupled fixed point results in cone metric spaces for $\tilde{\boldsymbol{w}}$-compatible mappings. Fixed Point Theory Appl. 2011, 27 (2011). doi:10.1186/1687-1812-2011-27

19. Aydi, H, Karapinar, E, Shatanawi, W: Coupled fixed point results for $(\psi, \bigotimes)$-weakly contractive condition in ordered partial metric spaces. Comput Math Appl. 62, 4449-4460 (2011). doi:10.1016/j.camwa.2011.10.021

20. Aydi, H, Postolache, M, Shatanawi, W: Coupled fixed point results for $(\psi, \bigotimes)$-weakly contractive mappings in ordered $G$ metric spaces. Comput Math Appl. 63, 298-309 (2012). doi:10.1016/j.camwa.2011.11.022

21. Aydi, H, Damjanović, B, Samet, B, Shatanawi, W: Coupled fixed point theorems for nonlinear contractions in partially ordered G-metric spaces. Math Comput Model. 54, 2443-2450 (2011). doi:10.1016/j.mcm.2011.05.059

22. Aydi, H, Abbas, M, Postolache, M: Coupled coincidence points for hybrid pair of mappings via mixed monotone property. J Adv Math Stud. 5(1):118-126 (2012)

23. Cho, YJ, Rhoades, BE, Saadati, R, Samet, B, Shatanawi, W: Nonlinear coupled fixed point theorems in ordered generalized metric spaces with integral type. Fixed Point Theory Appl. 2012, 8 (2012). doi:10.1186/1687-1812-2012-8

24. Karapinar, E: Couple fixed point on cone metric spaces. Gazi Univ J Sci. 24(1):51-58 (2011)

25. Karapinar, E: Coupled fixed point theorems for nonlinear contractions in cone metric spaces. Comput Math Appl. 59(12):3656-3668 (2010). doi:10.1016/j.camwa.2010.03.062

26. Sintunavarat, W, Cho, YJ, Kumam, P: Common fixed point theorems for c-distance in ordered cone metric spaces Comput Math Appl. 62, 1969-1978 (2011). doi:10.1016/j.camwa.2011.06.040 
27. Sintunavarat, W, Cho, YJ, Kumam, P: Coupled coincidence point theorems for contractions without commutative condition in intuitionistic fuzzy normed spaces. Fixed Point Theory Appl. 2011, 81 (2011). doi:10.1186/1687-1812-201181

28. Shatanawi, W, Samet, B, Abbas, M: Coupled fixed point theorems for mixed monotone mappings in ordered partial metric spaces. Math Comput Model. 55, 680-687 (2012). doi:10.1016/j.mcm.2011.08.042

29. Shatanawi, W: Coupled fixed point theorems in generalized metric spaces. Hacettepe J Math Stat. 40(3):441-447 (2011)

30. Shatanawi, W, Abbas, M, Nazir, T: Common coupled coincidence and coupled fixed point results in two generalized metric spaces. Fixed point Theory Appl. 2011, 80 (2011). doi:10.1186/1687-1812-2011-80

31. Shatanawi, W, Samet, B: On $(\psi, \mathbb{\Psi})$-weakly contractive condition in partially ordered metric spaces. Comput Math Appl. 62, 3204-3214 (2011). doi:10.1016/j.camwa.2011.08.033

32. Shatanawi, W: Fixed point theorems for nonlinear weakly C-contractive mappings in metric spaces. Math Comput Model. 54, 2816-2826 (2011). doi:10.1016/j.mcm.2011.06.069

33. Shatanawi, W: Partially ordered cone metric spaces and coupled fixed point results. Comput Math Appl. 60, 2508-2515 (2010). doi:10.1016/j.camwa.2010.08.074

34. Berinde, $\mathrm{V}$, Borcut, $\mathrm{M}$ : Tripled fixed point theorems for contractive type mappings in partially ordered metric spaces. Nonlinear Anal. 74(15):4889-4897 (2011). doi:10.1016/j.na.2011.03.032

35. Aydi, H, Karapinar, E, Postolache, M: Tripled coincidence point theorems for weak $\varphi$-contractions in partially ordered metric spaces. Fixed Point Theory Appl. 2012, 44 (2012). doi:10.1186/1687-1812-2012-44

36. Samet, B, Vetro, C: Coupled fixed point, f-invariant set and fixed point of N-order. Ann Funct Anal. 1(2):46-56 (2010)

37. Karapinar, E: Quartet fixed point for nonlinear contraction.http://arxiv.org/abs/1106.5472

38. Karapinar, E, Luong, NV: Quadruple fixed point theorems for nonlinear contractions. Comput Math Appl. doi:10.1016/j. camwa.2012.02.061

39. Karapinar, E: Quadruple fixed point theorems for weak 》-contractions. ISRN Mathematical Analysis 2011 (2011). Article ID 989423,16

40. Karapinar, E, Berinde, V: Quadruple fixed point theorems for nonlinear contractions in partially ordered metric spaces. Banach J Math Anal. 6(1):74-89 (2012)

41. Karapinar, E: A new quartet fixed point theorem for nonlinear contractions. JP J Fixed Point Theory Appl. 6(2):119-135 (2011)

42. Gohberg, I, Goldberg, S: Basic Operator Theory. Birkhäuser, Boston, MA (1981)

doi:10.1186/1687-1812-2012-71

Cite this article as: Mustafa et al: Mixed $g$-monotone property and quadruple fixed point theorems in partially ordered metric spaces. Fixed Point Theory and Applications 2012 2012:71.

\section{Submit your manuscript to a SpringerOpen ${ }^{\circ}$ journal and benefit from:}

- Convenient online submission

- Rigorous peer review

- Immediate publication on acceptance

- Open access: articles freely available online

- High visibility within the field

- Retaining the copyright to your article

Submit your next manuscript at $\gg$ springeropen.com 